\title{
Effet Du Stress Salin Sur Le Comportement Biochimique Et Anatomique Chez Deux Variétés De Piment (Capsicum Annuum L.) À Mila /Algérie
}

\section{Karima Bouassaba}

Centre Universitaire Abdelhafid Boussouf, Mila, Algérie, Département des Sciences de la Nature et de la Vie, Mila, Algérie, Faculté des Sciences de la Nature et de la Vie, Université Mentouri 1, Constantine, Algérie

\section{Saiida Chougui}

Laboratoire de Physiologie Végétale, Département de Biologie Université Mentouri1, Constantine, Algérie

Doi: 10.19044/esj.2018.v14n15p159 URL:http://dx.doi.org/10.19044/esj.2018.v14n15p159

Abstract

Soil salinity is a permanent threat to the survival of plants. The choice of a salt tolerant species could be a solution to this constraint. This paper focuses on the concept of adaptation and sensitivity during the vegetative phase of sweet pepper (Capsicum annuum L.) variety: Super marconi (Sp) and Marconi (M). Plants were stressed with different concentrations of $\mathrm{NaCl}$ ranging from $0,25,50$ to $150 \mathrm{mM} / 1 \mathrm{NaCl}$ for 60 days. The results obtained showed that the application of stress results in a moderate decrease in plant size and the content of Chlorophyll a, b which declined during times of stress. An increase in proline content and soluble sugars was recorded in the leaves. It could be an indicator of salinity tolerance which explains the maintenance of a good water status among the studied plants. Regarding the ionic content $\left(\mathrm{K}^{+}\right.$and $\left.\mathrm{Na}^{+}\right)$, salt stress caused a significant decrease in the content of $\mathrm{K}^{+}$, and a significant increase in $\mathrm{Na}^{+}$content in both pepper varieties. Concerning anatomical structure of stems, the action of combined salts $(50-150 \mathrm{Mmol} / \mathrm{L})$ causes a decrease in the size of the parenchymal cells, as well as the diameter of xylem vessels. It is concluded that the variety Super Marconi (Sp) is more tolerant to salinity compared to the variety Marconi (M). Hence, they have a significant role to play in agriculture, food, and economy.

Keywords: Tolerance, salinity, resistance, Capsicum anпиит L

\section{Résumé}

La salinité du sol est une menace permanente pour la survie des végétaux. Le choix d'une espèce tolérante au sel serait une solution à cette 
contrainte. Cette étude a porté sur le concept d'adaptation et de sensibilité pendant la phase végétative du poivron doux (Capsicum annuum L.) variété : Super marconi (Sp) et Marconi(M). Les plantes ont été stressées avec différentes concentrations de $\mathrm{NaCl}$ allant de $0,25,50$ à $150 \mathrm{mM} / 1$ de $\mathrm{NaCl}$ pendant 60 jours. Les résultats obtenus montrent que l'application du stress conduit à une diminution modérée de la taille des plantes et de la teneur en chlorophylle a, b qui diminue en période de stress. Une augmentation de la teneur en proline et des sucres solubles a été enregistrée au niveau des feuilles. Elle pourrait être un indice de tolérance de la plante à la salinité, ce qui explique le maintien d'un bon état hydrique parmi les plantes étudiées. Pour les teneurs ioniques $\left(\mathrm{K}^{+}\right.$et $\left.\mathrm{Na}^{+}\right)$, le stress salin a entrainé une diminution significative de la teneur en $\mathrm{K}^{+}$et une augmentation significative de la teneur en $\mathrm{Na}^{+}$chez les deux variétés de piment. Concernant les coupes anatomiques des tiges, l'action des sels combinés $(50-150 \mathrm{mMol} / \mathrm{L})$ engendre une diminution de la taille des cellules parenchymateuses, ainsi que celle du diamètre des vaisseaux de xylème. On conclue que la variété Super marconi (Sp) est plus tolérante à la salinité en comparaison avec la variété Marconi (M) et qu'elle a de l'importance dans l'agriculture, la nourriture et l'économie.

Mots-clé : Tolérance, salinité, résistance, Capsicum annuит L

\section{Introduction}

La salinité du sol et de l'eau constitue le problème majeur dans beaucoup des pays du monde. Elle est considérée comme le principal facteur abiotique qui limite la productivité végétale et le rendement agricole (Rozema \& Flower, 2008 ; Abd latef, 2010). Dans les écosystèmes arides et semi arides, la salinité résulte des fortes évaporations d'eau à partir du sol et d'une irrégulière et insuffisante pluviométrie.

Chaque année, les surfaces perdues à cause de la salinité des sols varient autour de 20 millions d'ha dans le monde. Ainsi que ces surfaces sont passées de 48 millions à 265 millions d'ha de terres agricoles touchées par la salinité, les surfaces agricoles affectées dans le monde seraient de 340 millions d'ha, soit 23\% des terres cultivées dans le monde (Cheverry, 1995). Selon Szabolcs (1994) un milliard d'ha est menacé dont 3,2 millions d'ha en Algérie sont affectés par la teneur excessive en sel (Sheng et al., 2008). Ce chiffre ne cesse pas d'augmenter d'une année à l'autre dû à la mauvaise qualité d'eau d'irrigation (Villa-Castorena et al., 2003), à l'intensification des cultures et à l'utilisation démesurée des fertilisants chimiques chez plusieurs espèces cultivées sous serre. Le poivron doux (Capsicum annuumL.) est considéré parmi les espèces légumières sensibles ou modérément sensibles à la salinité (Navarro et al., 2010). De nombreux auteurs (Chartzoulakis \& Klapaki, 2000; Zribi, 2009) ont remarqué que les doses de $\mathrm{NaCl}$ supérieur à $150 \mathrm{mM}$ réduisent 
fortement la croissance végétative et causent des symptômes de brulures et de toxicité.

Le stress salin est le résultat d'un déficit hydrique dans la plante sous forme de sécheresse physiologique (Mahajan \& Tuteja, 2005) .Ce stress osmotique se traduit essentiellement par l'accumulation toxique des ions dans les cellules et/ou un déséquilibre nutritionnel dû à un excès de certains ions (Souguir et al., 2013). En effet, selon le degré de stress dans le milieu, les plantes sont exposées à des modifications de leur comportement morphophysiologique, anatomique et biochimique (Nasir- Khan, 2010). Dans ces conditions, les plantes se trouvent en situation stressante et développent des mécanismes de défense (Denden et al., 2005). Parmi ces mécanismes, l'ajustement osmotique joue un rôle primordial dans la résistance ou la tolérance de la plante à un stress (Munns, 2002). La plante devra synthétiser des solutés organiques pour ajuster son potentiel hydrique. Une autre stratégie d'adaptation à la salinité consiste à synthétiser des osmoprotecteurs, principalement des composés aminés et des sucres, et à les accumulés dans le cytoplasme et les organites (Ashraf \& Foolad , 2007; Chen \& Jiang , 2010; Majumder et al., 2010). L'identification et la compréhension des mécanismes de tolérance des plantes à la salinité présentent l'amélioration variétale des plantes sensibles.

L'objectif du présent travail est d'étudier l'effet de différentes concentrations de la salinité sur la teneur en proline, sucres solubles, chlorophylle (a),(b), et les teneures ioniques $\left(\mathrm{K}^{+}\right.$et $\left.\mathrm{Na}^{+}\right)$chez les deux variétés de piment (Capsicum annuum L.), variété : Super marconi (Sp), Marconi (M).

\section{Matériels et Méthodes \\ Matériel \\ Végétal}

Cette étude est réalisée sur deux variétés de poivron doux Capsicum annuum L. var: Marconi (M)/ l'origine : chine) et Super marconi (Sp)/ l'origine : chine). L'expérimentation a été menée dans une chambre de culture au Centre Universitaire Abdelhafid Boussouf (Wilaya de Mila, Nord-Est Algérie.), durant 1'année 2016/2017.

\section{Le dispositif expérimental}

L'essai est conduit selon un dispositif expérimental en blocs complètement aléatoires (BAC), comportant 4 blocs (répétitions) de chaque variété de poivron doux (Marconi et Super marconi) ; pour chaque répétition deux plantes. 


\section{Les solutions salines}

Les plantes des deux variétés sont soumises aux différents traitements de $\mathrm{NaCl}$ :

$\mathrm{S}_{0}$ correspond à une concentration en

$\mathrm{NaCl}$ de $0 \mathrm{~m} \mathrm{Mol} / \mathrm{L}$ (témoin).

$\mathrm{S}_{1}$ correspond a une concentration en $\mathrm{NaCl}$ de $25 \mathrm{~m} \mathrm{Mol} / \mathrm{L}$.

$\mathrm{S}_{2}$ correspond a une concentration en $\mathrm{NaCl}$ de $50 \mathrm{~m} \mathrm{Mol} / \mathrm{L}$.

$\mathrm{S}_{3}$ correspond a une concentration en $\mathrm{NaCl}$ de $150 \mathrm{~m} \mathrm{Mol} / \mathrm{L}$.

\section{Les paramètres mesurés}

\section{Dosage des pigments chlorophylliens : chl $(\mathbf{a}, \mathbf{b})(\mathbf{m g} / \mathbf{g} / \mathbf{M F})$}

Dans un tube à essai à $100 \mathrm{mg}$ d'échantillon frais, coupé en petits fragments, sont ajoutés $10 \mathrm{ml}$ d'acétone à $95 \%$. L'ensemble est conservé à l'obscurité à $4^{\circ} \mathrm{C}$ pendant $48 \mathrm{~h}$. Le calcul des concentrations en chlorophylle a chl (a), chlorophylle b chl (b), s'effectue en appliquant diverses équations établies par (Lichtenthaler, 1987).

Chl a $\left(\mu \mathrm{g} . \mathrm{Ml}^{-1}\right)=12,25$. DO663 - 2,79. DO647

Chl b $\left(\mu \mathrm{g} . \mathrm{ml}^{-1}\right)=21,5$. DO647 - 5,10. DO663

Dosage des sucres solubles / glu (ug/100mg/MF)

Selon la méthode de Dubois (1956) concernant l'extraction des sucres solubles, $100 \mathrm{mg}$ de matériel végétal frais sont macérés dans $3 \mathrm{ml}$ d'éthanol pendant $48 \mathrm{~h}$. Après l'évaporation de l'alcool, $20 \mathrm{ml}$ d'eau sontajoutés.

\section{Dosage de la proline / Pro (ug/100mg/MF)}

D'après Nguyen and Roger (1987) le matériel végétal est étuvé et broyé (400 mg) avec $5 \mathrm{ml}$ d'éthanol à $95 \%$ auxquels sont rajoutés $15 \mathrm{ml}$ d'éthanol à $70 \%$. La solution finale est recueillie dans un tube à essai afin qu'elle soit décantée pendant $60 \mathrm{~min}$. Un volume de $5 \mathrm{ml}$ de la phase supérieure est prélevé puis additionné de $2 \mathrm{ml}$ de chloroforme et $3 \mathrm{ml}$ d'eau distillée. Après $24 \mathrm{~h}$ la phase supérieure est récupérée pour le dosage, réalisé selon la technique de (Bergman \& Loxley, 1970).

\section{Les teneurs en calcium et sodium $/ \mathrm{Na}^{+} / \mathrm{K}^{+}(\mathbf{p p m})$}

Afin d'analyser le comportement de transport et de stockage des ions au niveau de la plante, les concentrations des ions minéraux (sodium, potassium,) au niveau des feuilles ont été déterminées par un spectrophotomètre à flamme. Les plantes sont divisées en feuilles et racines. L'extraction est réalisée par le mélange des acides $\left(\mathrm{H}_{2} \mathrm{SO}_{4}-\mathrm{HClO}_{4-}\right.$ $\left.\mathrm{HNO}_{3} / 5: 2: 1\right)$ 


\section{Étude anatomique}

D'après Saadoun (2005) une réalisation des coupes transversales des tiges des deux variétés de piment (Capsicum annuum L.) doit être faite au moyen d'une lame de rasoir. Cette méthode repose sur l'utilisation de certains colorants: vert de méthyle, rouge Congo ou le carmino-vert de Mirande, ou bien vert de méthyle et carmin aluné. Elle permet de colorer exactement les parois cellulaires en fonction de leur composition chimique. suivantes:

Le principe de coloration des coupes levées repose sur les étapes

$>\quad$ Réalisation de coupes à la main à l'aide d'un simple rasoir ; l'organe est débitée en tranches de quelques micromètres d'épaisseur pour que les rayons lumineux puissent le traverser.

$>\quad$ Les coupes sont plongées, avant toute coloration, dans un bain d'hypochlorite de sodium à $12 \%$ pendant un quart d'heure pour évider le contenu des cellules.

$>\quad$ Rinçage à l'eau distillée.

$>\quad$ Immersion dans l'acide acétique à $2 \%$ pendant cinq minutes afin de fixer éventuellement les colorants sur les cellules et d'enlever toute trace d'hypochlorite de sodium.

$>\quad$ Rinçage sommaire à l'eau distillée.

$>\quad$ Immersion dans le double colorant (vert de méthyle / rouge Congo) pendant 10 à 15 minutes.

Rinçage à l'eau distillée, puis montage des coupes les plus fines entre lame et lamelle.

$>\quad$ Observation au microscope muni d'un appareil photo numérique (Motic).

\section{Analyse}

statistique

Le logiciel Excel Stat (2015) a été utilisé pour réaliser toutes les analyses de la variance ainsi que le test de New- man keuils $(\alpha=0.05)$ afin de comparer les moyennes entre les échantillons témoins et les traités pour chaque paramètre analysé.

\section{Résultats}

\section{Effet de salinité sur la teneur en chlorophylles}

Les teneurs en chlorophylle (a) et chlorophylle (b) ont été significativement réduites par l'effet de la salinité. Ainsi, chez le témoin, les teneures en chlorophylles sont restées plus importantes, comparativement aux teneurs en chlorophylles dosées chez les plantes traitées par $\mathrm{NaCl}(25,50$, et $150 \mathrm{mMol} / \mathrm{l}$. Les réductions les plus importantes ont été notées en présence de $150 \mathrm{~m} \mathrm{M} / \mathrm{L}$ de $\mathrm{NaCl}$ (Figure 1 et 2). En général, l'effet du sel s'est traduit 
chez les deux variétés de piment par une chute marquée des teneurs en chlorophylles. Cette chute a été plus prononcée chez la variété Super marconi pour la chlorophylle $\mathrm{b}\left(2.6-4.1 \mu \mathrm{g} \cdot \mathrm{ml}^{-1}\right)$,et la variété Marconi pour la chlorophylle a $\left(4.6-6.75 \mu \mathrm{g} \cdot \mathrm{ml}^{-1}\right)$.

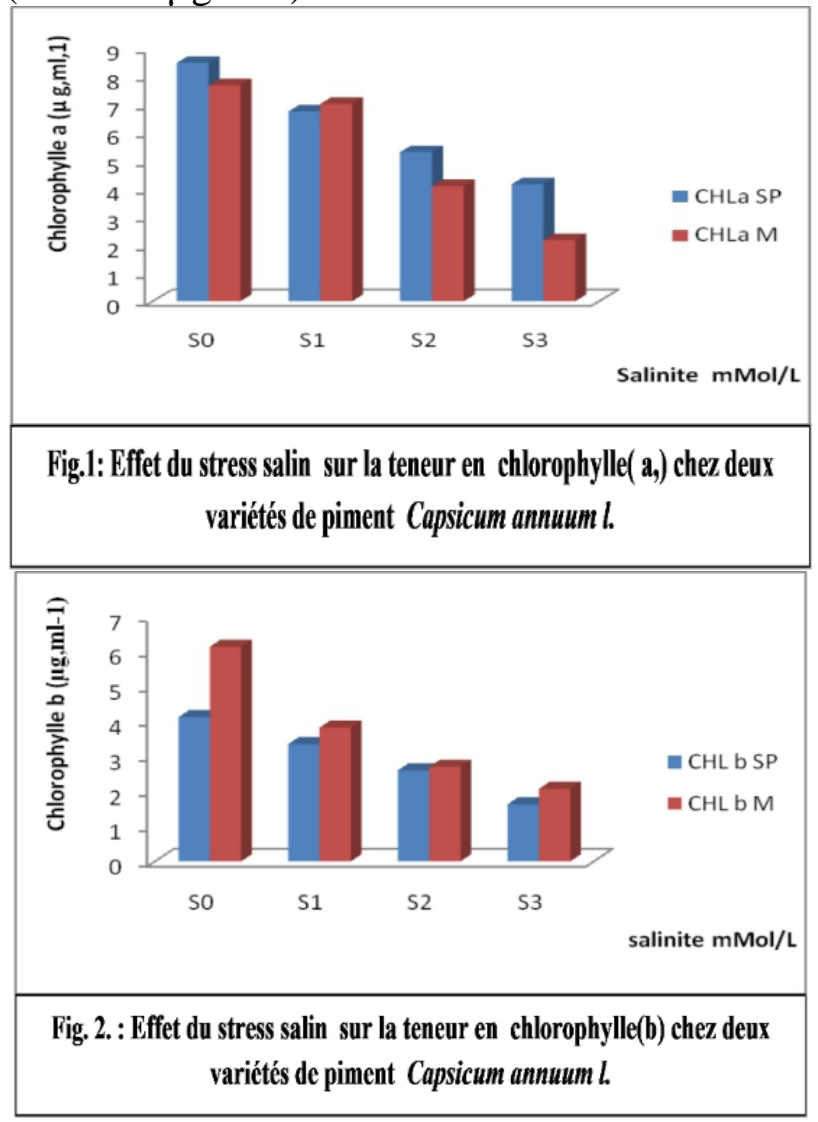

\section{Teneur en sucres solubles et proline}

Le sel a un effet significatif sur l'accumulation des sucres solubles $(\mathrm{p}<0.05)$. Les résultats indiquent que la corrélation est positive entre ces deux facteurs et hautement significative $(r=0.771, p<0.001)$ (Tableau 1$)$. D'après la Figure (3) la teneur en sucres solubles, sous contrainte saline, est très importante chez les deux variétés de piment ; elle passe de $\left(6.25 \mu \mathrm{g} \cdot \mathrm{ml}^{-1)} \mathrm{chez}\right.$ les plantes témoins à $(5.82)$ et $\left(6.64 \mu \mathrm{g} \cdot \mathrm{ml}^{-1)}\right.$ respectivement à $150 \mathrm{mM} / \mathrm{L}$ de $\mathrm{NaCl}$ chez super marconi et a (4.52 et $\left.5.29 \mathrm{~g} \mathrm{ml}^{-1}\right)$ chez marconi respectivement à $150 \mathrm{mM} / \mathrm{L}$ de $\mathrm{NaCl}$. 


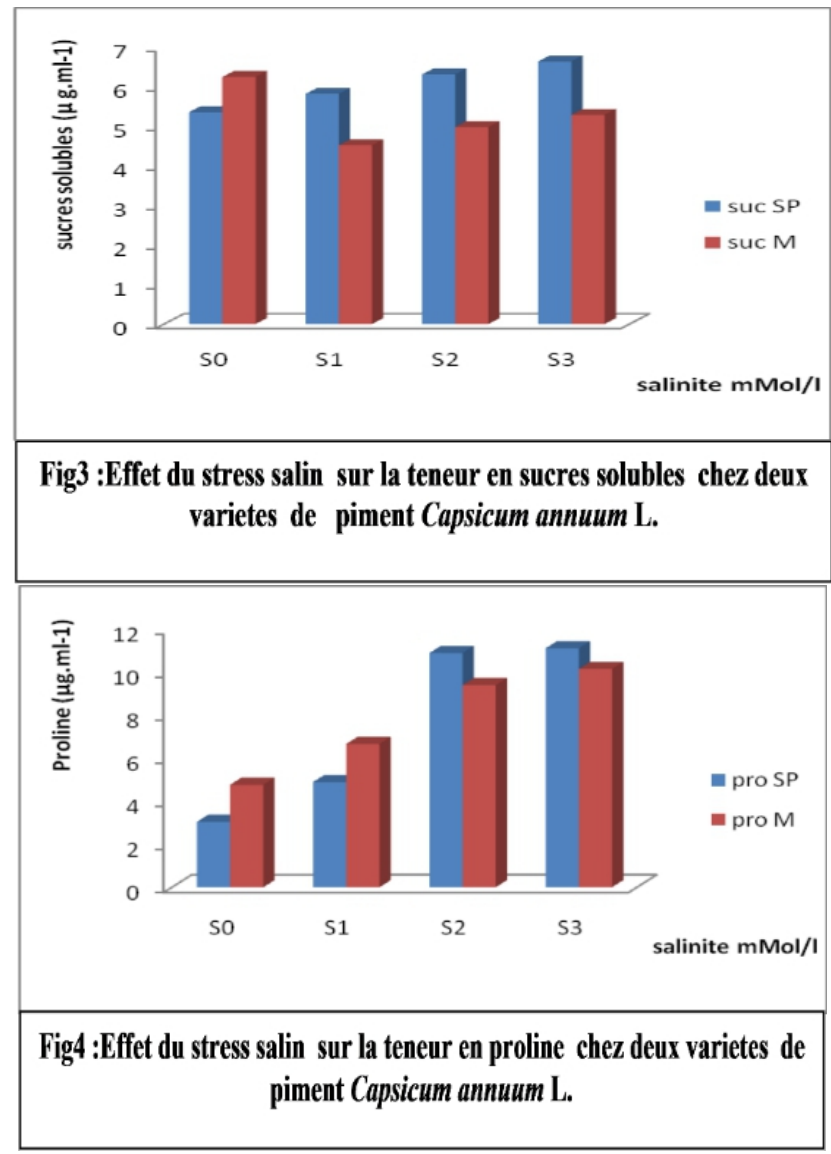

Concernant la proline (Figure 4) la salinité a un effet significatif sur l'accumulation de cet acide aminé $(p<0.005)$. Les résultats indiquent une corrélation positive et hautement significative entre la salinité et l'accumulation de la proline $(\mathrm{r}=0,686, \mathrm{p}<0.001)$ (Tableau1). En considérant la Figure (4), la proline évolue dans le même sens que la concentration de $\mathrm{NaCl}$. Elle varie significativement de $\left(3.04 \mu \mathrm{g} \cdot \mathrm{ml}^{-1}\right.$ à $7.76 \mu \mathrm{g} \cdot \mathrm{ml}^{-1)}$ respectivement pour les plantes témoins jusqu'à $\left(10.88 \mu \mathrm{g} \cdot \mathrm{ml}^{-1)}\right.$ et $(10.14$ $11.10 \mu \mathrm{g} . \mathrm{ml}^{-1)}$ pour les traitements $150 \mathrm{mMol} / \mathrm{L}$ de $\mathrm{NaCl}$.

\section{Teneur de sodium et potassium $\left(\mathrm{Na}^{+}, \mathrm{K}^{+}\right)$}

Les résultats obtenus révèlent une corrélation négative et hautement significative entre l'intensité du stress salin et les teneurs ioniques ( $r=-$ 0,$541 ; \mathrm{p}<0.001$ ) (Tableau 1). Le stress salin a provoqué une augmentation de la teneur en $\mathrm{Na}^{+}$chez les deux variétés étudiées. Les teneurs les plus faibles en $\mathrm{Na}^{+}$ont été enregistrées chez la variété super marconi, alors que celles les plus élevées sont affichées chez la variété Marconi . 


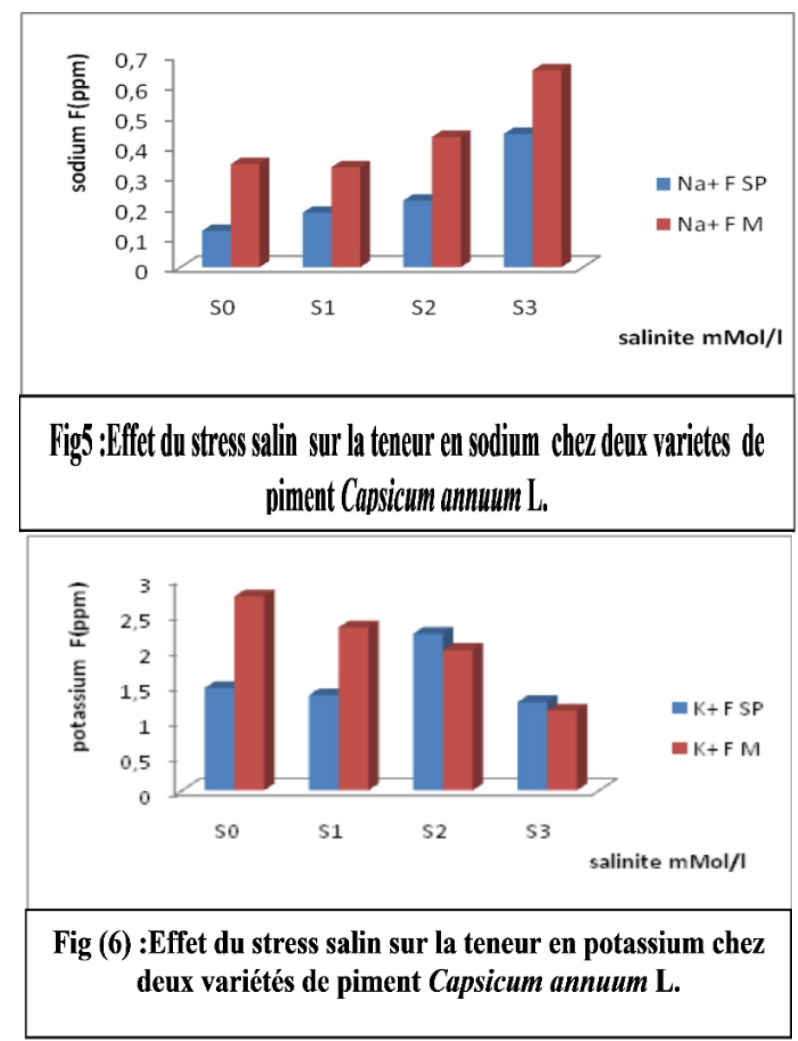

L'effet du traitement salin sur les concentrations en potassium varie selon les deux variétés (Figure 6). D'autre part, les concentrations de potassium baissent significativement au niveau de la tige de toutes les variétés sauf chez la variété ? qui maintient des concentrations identiques aux tolérantes marconi qui témoins. Les résultats indiquent que la corrélation positive entre ces deux facteurs est hautement significative $(r=0,579 \mathrm{p}<0.001$, Tableau 1). Les teneurs en $\mathrm{K}^{+}$sont plus élevées à $25 \mathrm{mmol} / \mathrm{L}$ de $\mathrm{NaCl}(2.31$ $2.75 \mathrm{ppm})$ par rapport aux traitements $150 \mathrm{mmol} / \mathrm{L}(1.99-2.22 \mathrm{ppm})$ et 150 $\mathrm{mmol} / \mathrm{L}(1.13-1.25-\mathrm{ppm})$. 
Tableau1 : Matrice de corrélation entre les paramètres biochimiques chez deux variétés de piment (Capsicum annuum L.) :

\begin{tabular}{|c|c|c|c|c|c|c|c|c|c|}
\hline Variables & TER & CHLA & CHLB & CHLT & SUC F & PS & PRO F & $\mathrm{Na}+\mathrm{F}$ & $k+F$ \\
\hline TER & 1 & & & & & & & & \\
\hline CHLA & $0,857^{* \star}$ & 1 & & & & & & & \\
\hline CHLB & $-0,736 * *$ & $0,752 * *$ & 1 & & & & & & \\
\hline CHLT & $-0,862 * *$ & $0,962^{* *}$ & $0,904^{* *}$ & 1 & & & & & \\
\hline SUC F & $0.771 * *$ & $-0,263$ & $-0,108$ & $-0,209$ & 1 & & & & \\
\hline PS & 0,076 & $0,851 * *$ & $0,847^{* *}$ & $0,903=*$ & $-0,158$ & 1 & & & \\
\hline PRO F & $0,686^{* *}$ & $-0,835^{* *}$ & $-0,742 * *$ & $-0,849 * *$ & 0,187 & $-0,765^{* *}$ & 1 & & \\
\hline $\mathrm{Na}+\mathrm{F}$ & $-0,541^{* *}$ & $-0,740^{* *}$ & $-0,301$ & $-0,606^{*}$ & 0,316 & $-0,503^{* *}$ & $0,531^{*}$ & $\mathbf{1}$ & \\
\hline $\mathbf{k}+\mathbf{F}$ & $0,579 * *$ & $0,457 *$ & $0,598^{* *}$ & $0,546^{*}$ & 0,069 & $0,585 * *$ & $-0,206$ & $-0,223$ & 1 \\
\hline
\end{tabular}

** :Significatif à $\mathrm{p} \leq 0.001$

* :Significatif à $\mathrm{p} \leq 0.05$

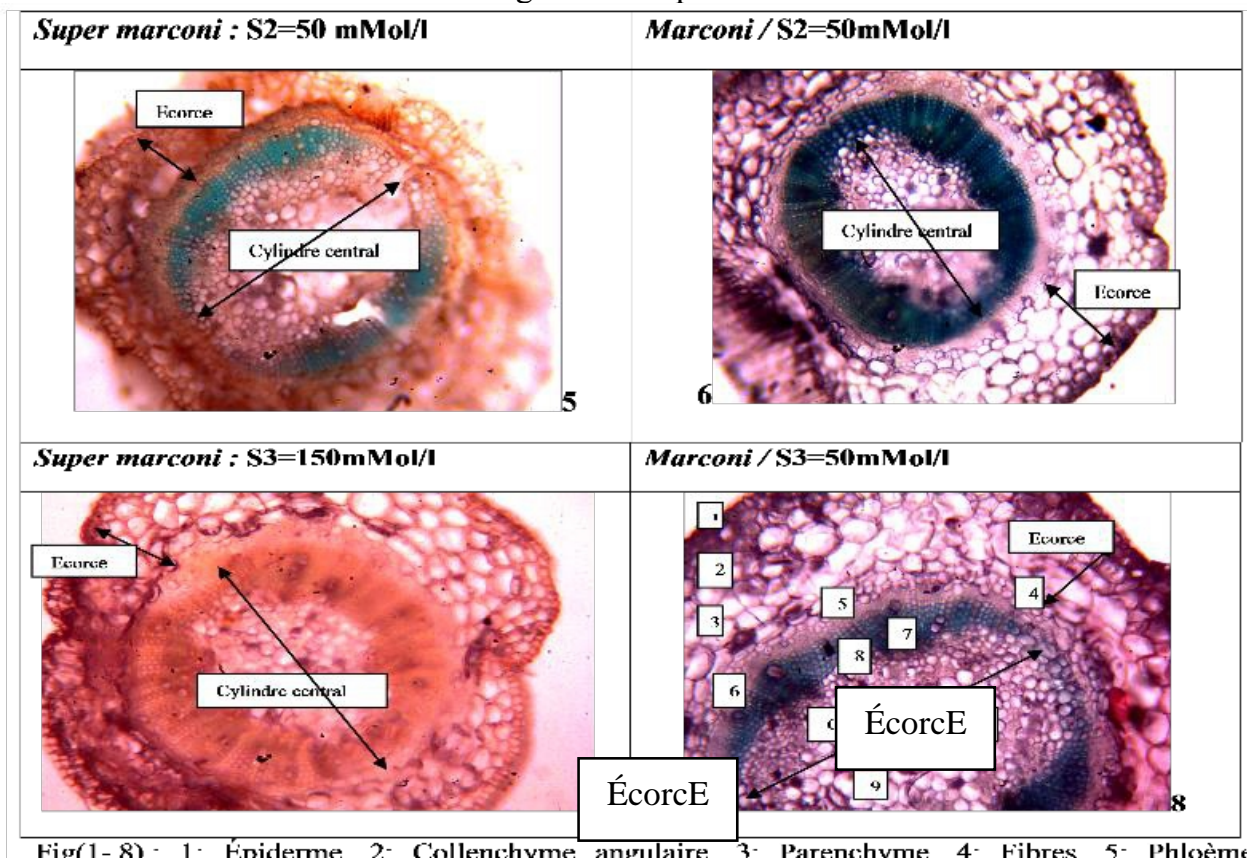
secondaire, 6: Cambium, 7: Phloème, 8: Xylème, 9: Moelle

Figure 7. Coupes transversales au niveau de la tige chez les deux variétés de piment (Capsicum annuum L.)

La comparaison des coupes anatomiques réalisées au niveau des tiges de piment (Capsicum annиum L.) des plantes témoins et stressées montre que la tige des plantes témoins est constituée de deux zones: l'écorce et le cylindre centrale (Figure 7). 
Chez les plantes de piment stressées, on observe une augmentation de la taille des cellules parenchymateuses sous le traitement salin de $25 \mathrm{mMol} / \mathrm{l}$, suivi d'une augmentation de l'épaisseur du parenchyme. Par contre sous le traitement de 50-150 mMol/1, l’épaisseur des cellules diminue.

\section{Discussion}

Pour s'adapter au stress salin, la plante peut éviter les dommages par la réduction de la croissance (Benmahioul et al., 2009) ; c'est l'effet le plus commun des stress abiotiques sur la physiologie des plantes. En effet, ce retard de développement permet à la plante d'accumuler de l'énergie et des ressources pour combattre le stress avant que le déséquilibre entre l'intérieur et l'extérieur de l'organisme n'augmente jusqu'à un seuil où les dommages seront irréversibles (Zhu, 2002).

\section{Teneurs en chlorophylles (a et b)}

La réduction de la chlorophylle (a) et chlorophylle (b) est peut être liée à la sensibilité de sa biosynthèse au chlorure de sodium. Ce dernier affecte moins la voie de biosynthèse de la chlorophylle(b) (Rhim et al., 2013). Le stress salin joue un rôle dans la diminution de l'activité de la chlorophylle et inhibe la synthèse de l'acide 5-amino-levulinique (Santos, 2004). Sous contraintes hydriques, il est constaté une perturbation au niveau des réactions photochimiques de la photosynthèse avec un blocage du transfert d'électrons entre LHCII et PSII (Neill et al., 2006). La diminution du taux du $\mathrm{CO}_{2}$ dans les feuilles est associée à une inhibition de la photosynthèse.

\section{Teneur en sucres solubles}

L'accumulation des sucres solubles chez les plantes de Capsicum annuum L. est toujours plus importante dans les tissus foliaires que dans les tiges. Les sucres solubles jouent un double rôle chez les plantes stressées, puisqu' ils participent aux événements métaboliques et à la déshydratation (Lepengue et al., 2012)., rôle qui est déterminant dans l'ajustement osmotique ainsi qu'au niveau de la stabilisation de certaines protéines (Bouatrous,2013). L'accumulation des sucres semble induire la gélification du contenu cellulaire en saturant le milieu intracellulaire; ce phénomène permet d'éviter la cristallisation des molécules contenues dans la cellule, et donc limite les dommages au niveau des structures cellulaires. L'accumulation des sucres est suggérée comme indice de résistance au stress salin (Muuns et al., 2006).

\section{Teneur en proline}

Chez les plantes du piment (Capsicum annuum L.) l'accumulation de la proline sous l'effet de la salinité se fait dans le sens des feuilles, aussi bien chez les plantes témoins que stressés; autrement dit cet acide aminé est 
concentré préférentiellement dans les feuilles à des teneurs significatives (Djerroudi, 2010). L'accumulation de proline chez les plantes stressées a une fonction de protection de la membrane cellulaire et participe à l'ajustement osmotique (Hassani et al., 2008). Elle agit en tant que composé soluble compatible sans exercer d'effet toxique comme le cas des ions (Silva-Ortega et al., 2008). En plus du rôle osmotique attribué à la proline, celle-ci intervient dans la détoxication des formes actives d'oxygène (Hong et al., 2000) et la stabilisation des protéines (Majumder et al., 2010).

\section{Teneur en $\mathrm{Na}^{+}$et $\mathrm{K}^{+}$}

En milieu salin, les plantes absorbent des quantités importantes de $\mathrm{Na}^{+}$ et de $\mathrm{Cl}^{-}$, mais le transport et l'accumulation de ces éléments semblent souvent dépendre du degré de tolérance de l'espèce considérée (Munns et al., 2006). La tolérance à la salinité ne se limite pas à une ? métabolique chez les plantes tolérantes (Larosa et al.,1991). Généralement, le $\mathrm{Na}^{+}$commence à avoir un effet inhibiteur sur l'activité enzymatique à partir d'une concentration de $100 \mathrm{mmol} / \mathrm{L}$. De la même manière, la capacité des plantes à réduire les teneurs en sodium dans le cytoplasme semble être un des éléments décisifs de la tolérance à la salinité (Yamaguchi \& Blumwald, 2006 ; Apse \& Blumwald, 2007).

Les espèces tolérantes et en particulier les halophytes accumulent des quantités importantes de sodium dans la partie aérienne, alors que les racines sont moins riches en $\mathrm{Na}^{+}$que les feuilles (Flowers \& Clomer, 2008 ; Atia et al., 2011). Au contraire, chez les glycophytes une limitation de la migration $\mathrm{du} \mathrm{Na}^{+}$vers les feuilles est présente, d'où des difficultés d'ajustement osmotique en milieu riche en sels et des troubles d'alimentation au niveau des racines. Presque la totalité de l'abaissement du potentiel osmotique est due à l'absorption du $\mathrm{Na}^{+}$chez les halophytes et les glycophytes (Hanana et al., 2011). La plupart des plantes excluent le $\mathrm{Na}^{+}$par les racines tandis que l'eau est captée par le sol (Munns, 2005).

Les observations montrent que le $\mathrm{Na}^{+}$reste prédominant dans les feuilles, alors que l'ion $\mathrm{K}^{+}$est légèrement présent avec des quantités plus ou moins importantes uniquement dans les feuilles des plantes témoins. Cette caractéristique ionique se rencontre généralement chez les espèces ou la teneur élevée en $\mathrm{K}^{+}$est liée à la tolérance à la salinité (Asins et al., 2012). Par contre, les concentrations en $\mathrm{K}^{+}$dans les feuilles des plantes témoins sont supérieures aux teneurs de $\mathrm{Na}^{+}$.

Selon Almeida et al. (2010), la tolérance à la salinité chez la tomate est souvent associée aux capacités particulières de maintenir une teneur élevée en $\mathrm{K}+$. L'efficacité d'absorption et d'utilisation du $\mathrm{K}^{+}$qui agit comme osmoticum, est donc capitale dans l'adaptation au stress salin (Hamraoui et al., 2011). Rapportant que les génotypes de piment tolérants au sel accumulent 
moins de $\mathrm{Na}^{+}$dans les feuilles que les génotypes ayant la capacité d'exclure $\mathrm{Na}^{+}$qui sont généralement plus tolérants à la salinité.

Les résultats de l'étude anatomique ont montré que tous les traitements salins sont capables d'induire des changements au niveau structural chez les deux variétés de piment. Anatomiquement, les résultats montrent que le diamètre des cellules des différents tissus change selon la concentration du sel. Ces résultats sont en accord avec ceux d'Akram et al. (2002) obtenus sur le blé soumis à une contrainte saline. Ces auteurs révèlent une diminution de la taille du parenchyme cortical et médullaire. Chez certaines plantes succulentes, la réaction est inversée, la surface des parenchymes cortical et médullaire augmente face au stress salin, phénomène qui a été observé chez le (Cynodon dactylon L.) (Hameed et al., 2010).

Ould el hadj-Khelil (2001) indique que chez des plantes de tomate soumises à un stress de $100 \mathrm{mM} / \mathrm{l}$ de $\mathrm{NaCl}$, de légères lésions apparaissent au niveau de la moelle des tiges et lorsque la concentration en sel passe à 200 $\mathrm{mM} / \mathrm{l}$, la moelle est fortement détruite. Un second changement concerne la diminution du diamètre des vaisseaux de xylème et l'augmentation de leur nombre sous les traitements $150 \mathrm{mMl} / 1$ de $\mathrm{NaCl}$.

\section{Conclusion}

La salinité est une contrainte majeure qui affecte la croissance et le développement des plantes surtout dans les régions arides et semi-arides qui souffrent des problèmes de salinisation des sols. Elle se manifeste au niveau de la plante entière à des degrés variables. La réponse varie d'un organe à un l'autre, d'une espèce à l'autre, selon la nature et l'intensité du stress.

Notre travail comprend l'étude de deux variétés de poivron doux (Capsicum annuum L.) à savoir Super marconi et Marconi sous des conditions salines pour connaitre la différence de leur réponse et déterminer la notion de la résistance et la sensibilité de la salinité.

Nous avons étudié des paramètres biochimiques dans les feuilles et durant la phase de croissance végétative de la plante.

Les résultats présentent des différences et des ressemblances entre les deux variétés durant les traitements salins. La variété Super marconi (Sup) est plus tolérante à la salinité comparée à la variété Marconi $(\mathrm{M})$. La salinité provoque une diminution des teneurs biochimiques en chloropylles (a), (b) et une accumulation des osmoprotecteurs tels que les sucres solubles et la proline surtout pour la concentration saline la plus sévère $(\mathrm{S} 3=150 \mathrm{mM} / \mathrm{l})$.

\section{References:}

1. Abd- Latef, A. (2010).Changes of antioxidative enzymes in salinity tolerance among different wheat cultvars.Cereal Res .Comm.38:43-55.

2. Akram, M., Ahmad, A., \& JANM ,M. (2002). The medicinal plants 
of salt Range. J. Biol. Sci., 2: 1035-1046.

3. Almeida, P.,Boer ,G., \& Boer ,A .(2014).Difference in shoot $\mathrm{Na}^{+}$ accumulation between two tomayo species are due to differences in ion affinity if HKTA1,2.J.PlantPhysiol.171,438-447

4. Apse, M.P., \& Blumwald, E. (2007). $\mathrm{Na}^{+}$transport in plants. FEBS Lett. 581(12) : 2247-2254.

5. 5-Ashraf, M, \& Asins, J.M.,Villata, L.,Aly, M.M.,Olias, R., Moralles, P.A., \& huertas, R. (2012).Two closely linked tomato HKT coding gene are positional $\mathrm{Na}^{+} / \mathrm{K}^{+}$homeostasis .Plantcell.Environ.36, 11711191.

6. Atia, A.,Smaoui ,A., Barhoumi Z., Abdelly C., \& DEBEZ A. (2011).Differential response to salinity andwater deficit stress in polypogon monospeliesis(L) Desf. Provenances during.Plant Biol, 13:541-600.

7. Benmahioul, B. F., Daguin, A., \& Kaid-Harche. (2009). Effet du stress salin sur la germination et la croissance in vitro du pistachier (Pistacia vera L.), Comptes Rendus Biologies, vol. 332, nº. 8, pp. 752758.

8. Bergman, I. \& Loxley, R.(1970). New spectrophotometric method for the determination of proline in tissue hydrolyzates," Anal. Chem., vol. 42, no.7, pp. 702-706.

9. Bouatrous, Y. (2013). Water stress correlated with senescence in durum wheat (Triticum durum Desf),"Advances in Environmental Biology, vol. 7, No.7, pp.1306-1314.

10. Chartzoulakis, K., \& Klapaki, G. (2000).Response of two greenhouse pepper hybrids to $\mathrm{NaCl}$ salinity during different growth stages.Sci.Hortic.86:247-260.

11. Chen, H., \& Jiang, J.G. (2010). Osmotic adjustment and plant adaptation to environmental changes related to drought and salinity. Environ. Rev. 18 (NA) : 309-319.

12. Denden M., T. Bettaieb, Alef, S., \& Mathlouthi, M. (2005). Effet de la salinité sur la fluorescence chlorophyllienne, la teneur en proline et la production florale de trois espèces ornementales," Tropicultura, vol $23, \mathrm{n}^{\circ} 4$, pp.220-225.

13. Djerroudi,Z.O., Belkhodja, M., Bissati ,S., \& Hadjadj, S. (2010). Effet du Stress Salin sur l'accumulation de Proline chez deux espèces d'Atriplex halimus L. et Atriplex canescens (Pursh) Nutt. European Journal of Scientific Research. vol.41, n², pp 249-260.

14. Dubois, M. K., Gilles, A., Hamilton, J. K., Rebers, P. A., \& Smith F. (1956). Colorimetric Method for Determination of Sugars and Related Substances," Anal. Chem., vol. 28, no.3, pp 350-356.

15. Flowes, T., \& Colmer, T.D. (2008). Salinity tolerance in halophytes 
.New phytol.179:945-963.

16. Foolad, M. R. (2007). Roles of glycine beanie and proline in improving plant abiotic stress resistance. Environ. Exp. Bot.59 (2) : 206-216

17. Hameed, M., Ashraf, M., Naz, N., \& Al-Qurainy, F.( 2010). Anatomical adaptations of Cynodon dactylon (L.) PERS., from the salt range PAKISTAN, to salinity stress. I. Root and stem anatomy.Pak. J. Bot., 42 (1): 279-289.

18. Hamrouni, L., Hanana , M., Abdelly, C., \& Ghorbel, A.(2011).Exclusion du chlorure et inclusion du sodium :deux mécanismes concomitants de tolérance à la salinité chez la vigne sauvage vitis vinifera Subsp.sylvestris(var.sejene)Bitechnol.Agronom.Soc.Znviron.15(13) : 387-400.

19. Hanana, M., Hamrouni, L., Cagnac, O., \& Blumwald, E. (2011). Mecanismes et strategies cellulairs de tolerance à la salinité $(\mathrm{NaCl})$ chez les plants .Environnemental Reviews. 19:121-140.

20. Hassani, A., Dellal A., Belkhodja, \& Kaid- Harche, M. (2008). Effet de la Salinité sur l'eau et certains osmolytes chez l'orge (HordeumVulgare L.). European Journal of scientific Research .vol.23.n⒈ PP. 61-69.22- James, R.A., \& Laüchli, A. (2006). Approaches to increasing the salt tolerance of wheat and other cereals. J. Exp Bot. 57(5) : 1025-1043.

21. Hong, Z., Lakkineni, K., Zhang, Z., \& Verma, D.P.S. (2000). Removal of feedback inhibition of D1-pyrroline-5-carboxylate synthetase results in increased proline accumulation and protection of plants from osmotic stress. Plant Physiol. 122(4) : 1129-1136.

22. Lepengue, A. N. I., Mouaragadja, B., Ibrahim, S., \& M'batchi. (2012). Réponse du maïs (Zea mays var. LG 60) au stress salin : étude de la synthèse de quelques composes biochimiques, "Journal of Animal \& Plant Sciences, vol. 14, ${ }^{\circ} 1$, pp. 1866-1872.

23. Lichtenthaler, H.K. (1987). Chlorophyllsand carotenoids: Pigments of photosynthetic. biomembranes Methods in Enzymology, vol. 148, pp. 350-382.

24. Larosa, P.C., Rhodes, D., Rhodes, P.M., Bressan, R.A., \& Csonka, L.N. (1991). Elevated accumulation of proline in NaCl-adapted tobacco cells is not due to altered D1pyrroline-5-carboxylate reductase. Plant Physiol. 96(1) : 245-250.

25. Mahajan, S., \&. Tuteja, N. (2005). Cold salinity and drought stresses, An overview Archives of Biochemistry and Biophysics, vol. 444, $\mathrm{n}^{\circ} 2$, pp. 139-158.

26. Majumder , A. L., Sengupta, S., \& Goswani, L. (2010). Osmolyte regulation in abiotic stress. Chap dans Abiotic stress adaptation in 
plants : Physiological, molecular and genomic foundation. Sous la direction de A. Pareek, S.K. Sopory, H.J. Bohmert et Govindjee.p.349370.

27. Munns, R. (2002). Comparative physiology of salt and water stress. Plant, Cell and Environment 25, 239-250.

28. Munns, R. (2005).Genes and salt tolerance: bringing them together. New Phytol.167(3) : 645-663.

29. Munns, R. A., James, A., \& Lauchli (2006). Approaches to increasing the salt tolerance of wheat and other cereals, Journal of Experimental Botany, vol. 57, $\mathrm{n}^{\circ}$ 5, pp. 1025-1043.

30. Nasir- Khan, M. H., Siddiqui, F., Mohammad, M., Naeem, M., \& Masroor, A. K. (2010). Calcium chloride and gibberellic acid protect linseed (Linum usitatissimum L.) from $\mathrm{NaCl}$ stress by inducing antioxidative defense system and osmoprotectant accumulation, "Acta Physiologiae Plantarum, vol. 32, No. 1, pp. 121-132.

31. Navarro, J.M., Garrido, C., Flores, P., \& Martinez, V. (2010). The effect of salinity on yield and fruit quality of pepper grown in perlite. Spanish Journal of Agricultural Research, 8, 142-150.

32. Nguyen, S.T. \& Roger, P. (1971). Methodes g'extraction et de purification des acides amines libres et des proteins de tissus végétaux, journal of Chromatography A., vol.61pp.349-351.

33. Ould El hadj, K. A. (2001). Contribution à l'étude de réponses métaboliques de la tomate la salinité Thèse de doctorat en Science de la Vie et de l'Environnement, Université de Rennes-I (France). 117 P

34. R'him, T. I., Tlili, I., Hnan, R., Ilahy, A., Benali \& Jebari, H. (2013). Effet du stress salin sur le comportement physiologique et métabolique de trois variétés de piment (Capsicum annuum 1.), Journal of Applied Biosciences, vol.66, pp.5060-5069.

35. Rozema, J. \& Flowers, T. (2008). Crops for a salinized world. Science 322, 1478-1480.

36. Santos, C. V. (2004). Regulation of chlorophyll biosynthesis and degradation by salt stress in sunflower leaves, Scientia Horticulturae, vol. 103, n 1 , pp. 93-99.

37. Sheng, M., Tang, M., Chan, H., Yang, B., Zhang, F., \& Huang, Y. (2008). Influence of arbuscular mycorrhiza on photosynthesis and water status of maize plants under salt stress. Mycorrhiza 18, 287-296.

38. Silva-Ortega, C.O., Ochoa-Alfaro, A.E., Reyes-Aguero, J.A., AguadoSantacruz, G.A., \& Jimenez-Bremont, J.F. (2008). Salt stress increases the expression of $\mathrm{p} 5 \mathrm{cs}$ gene and induces proline accumulation in cactus pear. Plant Physiol. Biochem. 46(1) : 82-92.

39. Souguir, D., Jouzdan, O., Khouja, M.L., \& Achicha, M. (2003). Suivi de la croissance d'Aloe vera en milieu salin : Prcelle de kalaàt 
Landelous (Tunisie),'Etude et Gestion des Sols, vol. 30, no.3, pp. 1926.

40. Villa-Castorena, M., Ulery, A.L., Catalan-Valencia, E.A., \& Remmenga, M.D. (2003). Salinity and nitrogen rate effects on the growth and yield of Chile pepper plants. Soil.Sci. Soc. Am. J. 67, 17811789.

41. Yamaguchi, T., \& Blumwald, E. (2006). Developing salt-tolerant crop plants: challenges and opportunities. Trends Plant Sci. 10(12) : 615620.

42. Zhu, J.K. (2003). Regulation of ion homeostasis under salt stress. Curr. Opin. Plant Biol.6(5):445.

43. Zribi, L., Gharbi, F., Rezgui, F., Rejeb, S., Nahdi, H., \& Rejeb, M.N. ( 2009). Application of chlorophyll fluorescence for the diagnosis of salt stress in tomato "Solanum lycopersicum (variety Rio Grande)". Scientia Horticulturae 120, 367-372. 